\title{
In Situ and Ex Situ Electron Microscopy and Spectroscopy Investigation of Capacity Fade Mechanisms of Rechargeable Li-S Batteries
}

\author{
V. P. Oleshko, C. Scordilis-Kelley and A. Xiao
}

Sion Power, Tucson AZ 85756-7129

The development of high performance Li-based batteries for emerging applications requires understanding and improving the behavior of electrode/electrolyte interfaces. Direct live in situ observations of batteries cycled within a SEM combined with indirect methods such as electrochemical impedance spectroscopy (EIS) and galvanostatic (GS) methods are promising to determine the interface microstructure/composition and active surface area evolution upon cycling. This may allow one to establish a link between interface properties, performance and electrode defects. Sion Power high-energy density rechargeable Li-S battery technology already offers twice the energy density $(350 \mathrm{Wh} / \mathrm{kg})$ of the best commercial Li-ion batteries and it is of interest in powering of all-electrical vehicles and long-flying unmanned aircrafts $[1,2]$. Novel approaches to the $3 \mathrm{D}$ design of cells followed by testing and structural characterization under varying processing conditions $(\mathrm{T}, \mathrm{P})$ become crucial for further improving in the cycle life. Here we report SEM/EDS spectral imaging (SI) study of composite sulfur-carbon cathodes under pressure in situ complemented by ex situ (FE)SEM/BSE/EDS and EIS/GS evaluation of cathodes in secondary Li-S batteries subjected to cycling without and under pressure.

In situ structural investigation of porous sulfur-carbon cathodes under compression (FIG. 1). An ultra-precision compression loading set-up enables characterizing composite cathodes by SEM/EDS SI in situ under desirable pressure in the range of $0-30 \mathrm{~kg} / \mathrm{cm}^{2}$. Thickness reduction by 17-22.2 $\mu \mathrm{m}$ under chosen conditions was revealed by in situ SEM. Compression could result in cathode densification and affect shapes/sizes of micro- and mesopores, aggregated carbon structures and 10-50 $\mu \mathrm{m}$ sulfur segregations.

Ex situ high-resolution (FE)SEM/BSE/EDS studies of cycled sulfur-carbon cathodes (FIG. 2) and related analyses of compression effects on cycled Ni foam cathodes (FIG. 3) have been performed using a hermetic holder designed for microstructural characterization of environmentally sensitive samples. Conventional and high-resolution (FE) SEM of cycled composite cathodes complemented by EDS X-ray SI analyses indicated drastic morphology changes induced by deposited $\mathrm{Li}_{2} \mathrm{~S}$. 3D SEM observations demonstrated a variety of morphologies, including dense $\mathrm{Li}_{2} \mathrm{~S}$ deposits over aggregated carbon particles and $\mathrm{Ni}$ foam supports, $\mathrm{Li}_{2} \mathrm{~S}$ precipitates filling inter space between carbon aggregates and unfilled or partially filled micropores and mesopores. Multivariate statistical analysis of EDS X-ray SI data indicated preferential accumulation of sulfur and to some extent of carbon and oxygen-containing phases in deposited areas. EIS confirmed reduction in interface build up with cycling under pressure. Demonstrated findings are involved both in the improvement of the reliability of assembling and enhancement of battery cycling performances. Such achievements constitute the technical platform for our near future targets namely the live in situ SEM/EIS/GS analyses of sectioned Li$\mathrm{S}$ batteries cycled within the microscope.

References

[1] Y. Mikhaylik et al., ECS Transactions, 13 (19) (2008) 53.

[2] http://news.bbc.co.uk/go /pr/fr/-/2/hi/science/nature/7577493.stm. Published: 2008/08/24 04:21:42 GMT. 


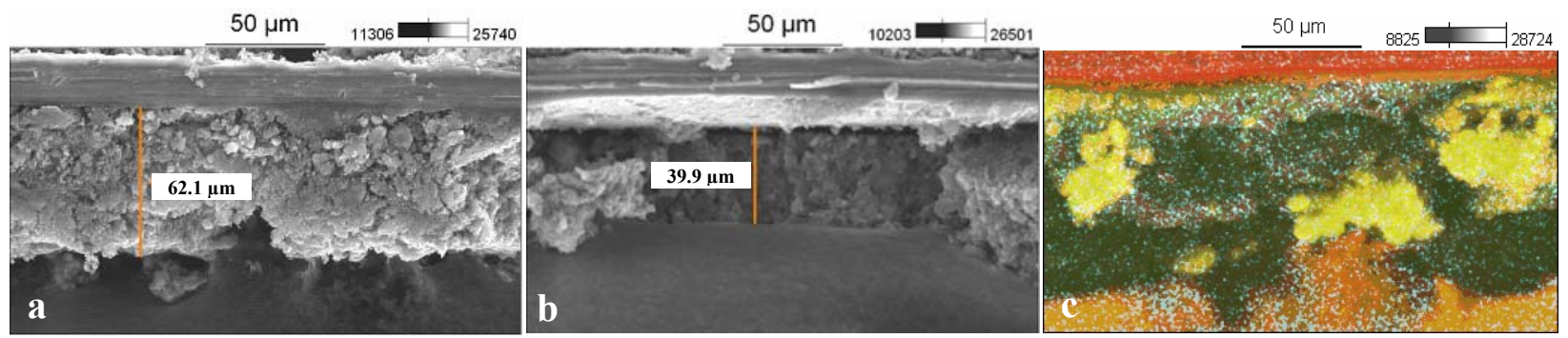

FIG. 1. Cross-sections of a carbon-sulfur cathode, SEM, secondary electrons (SE): (a) as received, (b) in situ under pressure; (c) in situ, compressed cathode, X-ray SI composite overlay of SK (yellow), CK (orange), OK (cyan) and AlK (red) X-ray maps showing Al alloy current collector (top) and 10-50 $\mu \mathrm{m}$ sulfur-enriched segregations.

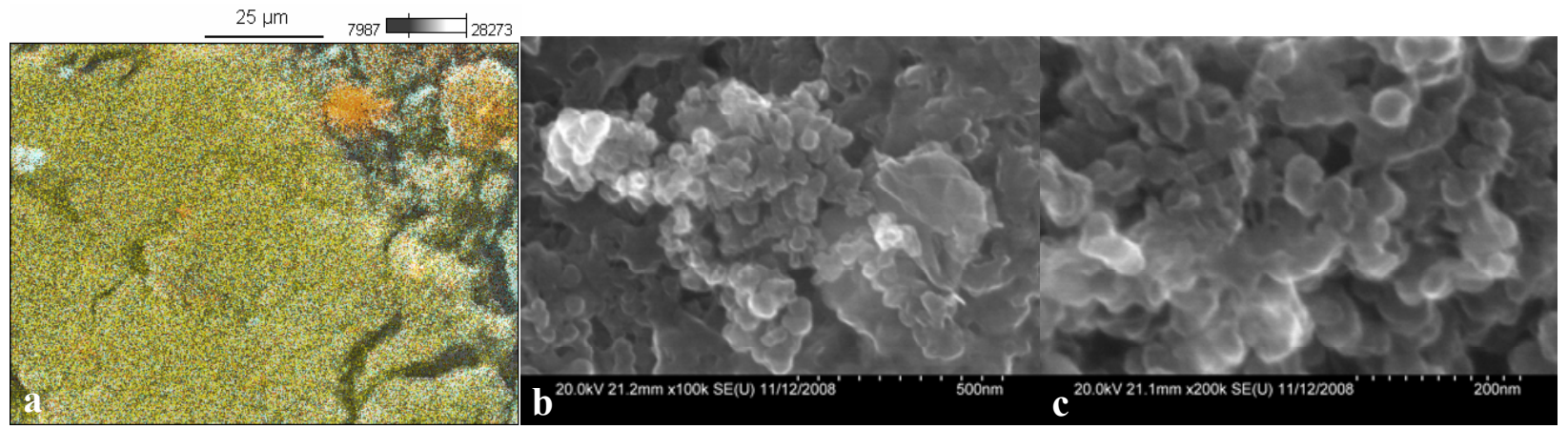

FIG. 2. Dense $\mathrm{Li}_{2} \mathrm{~S}$ deposits on cycled sulfur-carbon cathode, $142^{\text {nd }}$ charge, HR-FESEM, SE: (a) X-ray SI composite overlay of SK (yellow), CK (orange) and OK (cyan) X-ray maps; (b, c) $\mathrm{Li}_{2} \mathrm{~S}$ precipitating over carbon aggregates and filling inter space between particles.
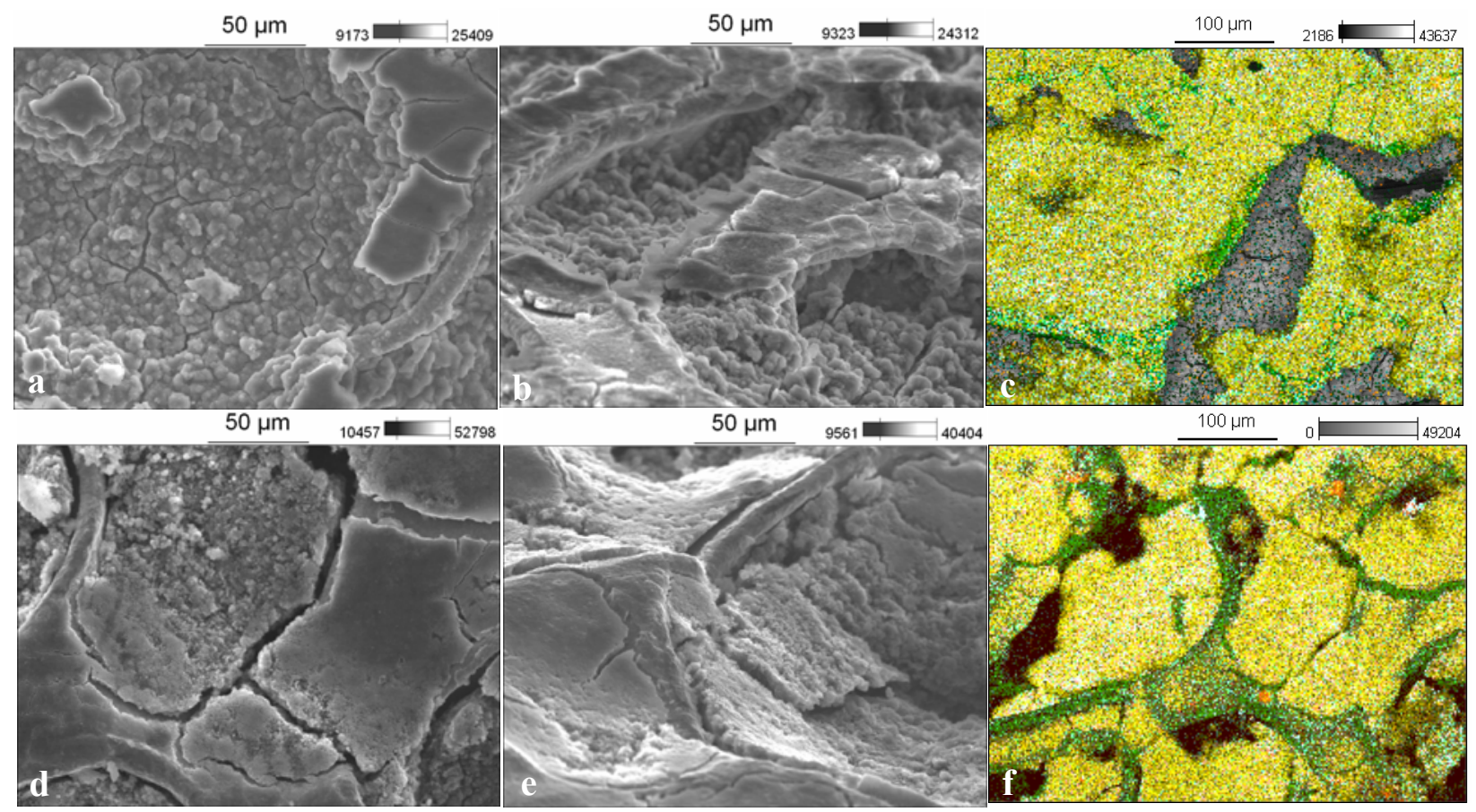

FIG. 3. Ni foam cathodes cycled without (upper row) and under pressure (bottom row). $\mathrm{Li}_{2} \mathrm{~S}$ deposits on carbon aggregates and Ni foam, SE: (a, d) plane view; (b, e) $60^{\circ}$ tilt view; (c, f) Xray SI composite overlays of SK (yellow), CK (orange), OK (cyan) and NiK (green) X-ray maps. 